\title{
Existence of positive periodic solutions for third-order nonlinear delay differential equations with variable coefficients
}

\author{
Abdelouaheb Ardjouni*, Ahcene Djoudi
}

ABstract. In this paper, the following third-order nonlinear delay differential equation with periodic coefficients

$$
\begin{aligned}
& x^{\prime \prime \prime}(t)+p(t) x^{\prime \prime}(t)+q(t) x^{\prime}(t)+r(t) x(t) \\
= & f(t, x(t), x(t-\tau(t)))+\frac{d}{d t} g(t, x(t-\tau(t))),
\end{aligned}
$$

is considered. By employing Green's function and Krasnoselskii's fixed point theorem, we state and prove the existence of positive periodic solutions to the third-order nonlinear delay differential equation.

\section{INTRODUCTION}

Third order differential equations arise from in a variety of different areas of applied mathematics and physics, as the deflection of a curved beam having a constant or varying cross section, three layer beam, electromagnetic waves or gravity driven flows and so on $[24,29]$.

Delay differential equations have received increasing attention during recent years since these equations have been proved to be valuable tools in the modeling of many phenomena in various fields of science and engineering, see the monograph [12, 26] and the papers [1]-[23], [25] [27]-[29], [31]-[34] and the references therein.

The second order nonlinear delay differential equation with periodic coefficients

$$
\begin{gathered}
x^{\prime \prime}(t)+p(t) x^{\prime}(t)+q(t) x(t)= \\
=f(t, x(t), x(t-\tau(t)))+\frac{d}{d t} g(t, x(t-\tau(t))),
\end{gathered}
$$

2010 Mathematics Subject Classification. Primary: 34K13, 34A34; Secondary: Secondary 34K30, 34L30.

Key words and phrases. Fixed point, positive periodic solutions, third-order delay differential equations.

Full paper. Received 28 February 2019, accepted 6 September 2019, available online 2 October 2019. 
has been investigated in [9]. By using Krasnoselskii's fixed point theorem and the contraction mapping principle, Ardjouni and Djoudi obtained existence and uniqueness of periodic solutions.

In [29], Ren, Siegmund and Chen discussed the existence of positive periodic solutions for the third-order differential equation

$$
x^{\prime \prime \prime}(t)+p(t) x^{\prime \prime}(t)+q(t) x^{\prime}(t)+r(t) x(t)=g(t, x(t)) .
$$

By employing the fixed point index, the authors obtained existence results for positive periodic solutions.

Inspired and motivated by the works mentioned above and the papers [1]-[23], [25], [27]-[29], [31]-[34] and the references therein, we concentrate on the existence of positive periodic solutions for the third-order nonlinear delay differential equation

$$
\begin{gathered}
x^{\prime \prime \prime}(t)+p(t) x^{\prime \prime}(t)+q(t) x^{\prime}(t)+r(t) x(t) \\
=f(t, x(t), x(t-\tau(t)))+\frac{d}{d t} g(t, x(t-\tau(t))),
\end{gathered}
$$

where $p, q, r, \tau$ are continuous real-valued functions. The functions $g$ : $\mathbb{R} \times \mathbb{R} \rightarrow \mathbb{R}$ and $f: \mathbb{R} \times \mathbb{R} \times \mathbb{R} \rightarrow \mathbb{R}$ are continuous in their respective arguments. To show the existence of positive periodic solutions, we transform (1) into an integral equation and then use Krasnoselskii's fixed point theorem. The obtained integral equation splits in the sum of two mappings, one is a contraction and the other is compact.

The organization of this paper is as follows. In section 2, we introduce some notations and lemmas, and state some preliminary results needed in later section, then we give the Green's function of (1), which plays an important role in this paper. In section 3 , we present our main results on existence of positive periodic solutions of (1).

We state Krasnoselskii's fixed point theorem which enables us to prove the existence of positive periodic solutions to (1). For its proof we refer the reader to [30].

Theorem 1.1 (Krasnoselskii). Let $\mathbb{M}$ be a closed convex nonempty subset of a Banach space $(\mathbb{B},\|\cdot\|)$. Suppose that $H_{1}$ and $H_{2}$ map $\mathbb{M}$ into $\mathbb{B}$ such that

(i) $x, y \in \mathbb{M}$, implies $H_{1} x+H_{2} y \in \mathbb{M}$,

(ii) $H_{1}$ is compact and continuous,

(iii) $\mathrm{H}_{2}$ is a contraction mapping.

Then there exists $z \in \mathbb{M}$ with $z=H_{1} z+H_{2} z$.

In this paper, we give the assumptions as follows that will be used in the main results. 
(h1) There exist differentiable positive $T$-periodic functions $a_{1}$ and $a_{2}$ and a positive real constant $\rho$ such that

$$
\left\{\begin{array}{l}
a_{1}(t)+\rho=p(t), \\
a_{1}^{\prime}(t)+a_{2}(t)+\rho a_{1}(t)=q(t), \\
a_{2}^{\prime}(t)+\rho a_{2}(t)=r(t) .
\end{array}\right.
$$

(h2) $p, q, r, \tau \in C\left(\mathbb{R}, \mathbb{R}^{+}\right)$are $T$-periodic functions with $\tau(t) \geq \tau^{*}>0$ and

$$
\int_{0}^{T} p(s) d s>\rho T, \int_{0}^{T} q(s) d s>0 .
$$

(h3) The functions $g(t, x)$ and $f(t, x, y)$ are continuous $T$-periodic in $t$ and continuous in $x$ and in $x$ and $y$, respectively.

\section{GREEN's FUnCTION OF THIRD-ORDER DIFFERENTIAL EQUATION}

For $T>0$, let $P_{T}$ be the set of all continuous scalar functions $x$, periodic in $t$ of period $T$. Then $\left(P_{T},\|\|.\right)$ is a Banach space with the supremum norm

$$
\|x\|=\sup _{t \in \mathbb{R}}|x(t)|=\sup _{t \in[0, T]}|x(t)|
$$

We consider

$$
x^{\prime \prime \prime}(t)+p(t) x^{\prime \prime}(t)+q(t) x^{\prime}(t)+r(t) x(t)=h(t),
$$

where $h$ is a continuous $T$-periodic function. Obviously, by the condition $(h 1),(2)$ is transformed into

$$
\left\{\begin{array}{l}
y^{\prime}(t)+\rho y(t)=h(t), \\
x^{\prime \prime}(t)+a_{1}(t) x^{\prime}(t)+a_{2}(t) x(t)=y(t) .
\end{array}\right.
$$

Lemma 2.1 ([7]). If $y, h \in P_{T}$, then $y$ is a solution of equation

$$
y^{\prime}(t)+\rho y(t)=h(t)
$$

if only if

$$
y(t)=\int_{t}^{t+T} G_{1}(t, s) h(s) d s
$$

where

$$
G_{1}(t, s)=\frac{\exp (\rho(s-t))}{\exp (\rho T)-1} .
$$

Corollary 2.1. Green function $G_{1}$ satisfies the following properties

$$
\begin{gathered}
G_{1}(t+T, s+T)=G_{1}(t, s), \quad G_{1}(t, t+T)=G_{1}(t, t) \exp (\rho T), \\
G_{1}(t+T, s)=G_{1}(t, s) \exp (-\rho T), \quad G_{1}(t, s+T)=G_{1}(t, s) \exp (\rho T), \\
\frac{\partial}{\partial t} G_{1}(t, s)=-\rho G_{1}(t, s),
\end{gathered}
$$




$$
\frac{\partial}{\partial s} G_{1}(t, s)=\rho G_{1}(t, s),
$$

and

$$
m_{1} \leq G_{1}(t, s) \leq M_{1},
$$

where

$$
m_{1}=\frac{1}{\exp (\rho T)-1}, \quad M_{1}=\frac{\exp (\rho T)}{\exp (\rho T)-1} .
$$

Lemma 2.2 ([28]). Suppose that (h1) and (h2) hold and

$$
\frac{R_{1}\left[\exp \left(\int_{0}^{T} a_{1}(v) d v\right)-1\right]}{Q_{1} T} \geq 1,
$$

where

$$
\begin{gathered}
R_{1}=\max _{t \in[0, T]}\left|\int_{t}^{t+T} \frac{\exp \left(\int_{0}^{T} a_{1}(v) d v\right)}{\exp \left(\int_{0}^{T} a_{1}(v) d v\right)-1} a_{2}(s) d s\right|, \\
Q_{1}=\left(1+\exp \left(\int_{0}^{T} a_{1}(v) d v\right)\right)^{2} R_{1}^{2} .
\end{gathered}
$$

Then there are continuous T-periodic functions $a$ and $b$ such that

$$
b(t)>0, \quad \int_{0}^{T} a(v) d v>0,
$$

and

$$
a(t)+b(t)=a_{1}(t), b^{\prime}(t)+a(t) b(t)=a_{2}(t), \text { for } t \in \mathbb{R} .
$$

Lemma 2.3 ([32]). Suppose the conditions of Lemma 2.2 hold and $y \in P_{T}$. Then the equation

$$
x^{\prime \prime}(t)+a_{1}(t) x^{\prime}(t)+a_{2}(t) x(t)=y(t),
$$

has a $T$ periodic solution. Moreover, the periodic solution can be expressed by

$$
x(t)=\int_{t}^{t+T} G_{2}(t, s) y(s) d s,
$$

where

$$
\begin{aligned}
G_{2}(t, s) & =\frac{\int_{t}^{s} \exp \left[\int_{t}^{v} b(u) d u+\int_{v}^{s} a(u) d u\right] d v}{\left[\exp \left(\int_{0}^{T} a(v) d v\right)-1\right]\left[\exp \left(\int_{0}^{T} b(v) d v\right)-1\right]} \\
& +\frac{\int_{s}^{t+T} \exp \left[\int_{t}^{v} b(u) d u+\int_{v}^{s+T} a(u) d u\right] d v}{\left[\exp \left(\int_{0}^{T} a(v) d v\right)-1\right]\left[\exp \left(\int_{0}^{T} b(v) d v\right)-1\right]}
\end{aligned}
$$


Corollary 2.2. Green's function $G_{2}$ satisfies the following proprieties

$$
\begin{aligned}
G_{2}(t+T, s+T) & =G_{2}(t, s), G_{2}(t, t+T)=G_{2}(t, t), \\
G_{2}(t+T, s) & =\exp \left(-\int_{0}^{T} b(v) d v\right) \\
& \times\left[G_{2}(t, s)+\int_{t}^{t+T} E(t, u) F(u, s) d u\right], \\
\frac{\partial}{\partial t} G_{2}(t, s) & =-b(t) G_{2}(t, s)+F(t, s), \\
\frac{\partial}{\partial s} G_{2}(t, s) & =a(t) G_{2}(t, s)-E(t, s),
\end{aligned}
$$

where

$$
E(t, s)=\frac{\exp \left(\int_{t}^{s} b(v) d v\right)}{\exp \left(\int_{0}^{T} b(v) d v\right)-1}, F(t, s)=\frac{\exp \left(\int_{t}^{s} a(v) d v\right)}{\exp \left(\int_{0}^{T} a(v) d v\right)-1} .
$$

Lemma $2.4([28])$. Let $A=\int_{0}^{T} a_{1}(v) d v, B=T^{2} \exp \left(\frac{1}{T} \int_{0}^{T} \ln \left(a_{2}(v)\right) d v\right)$. If

$$
A^{2} \geq 4 B
$$

then

$$
\begin{aligned}
& \min \left\{\int_{0}^{T} a(v) d v, \int_{0}^{T} b(v) d v\right\} \geq \frac{1}{2}\left(A-\sqrt{A^{2}-4 B}\right)=l, \\
& \max \left\{\int_{0}^{T} a(v) d v, \int_{0}^{T} b(v) d v\right\} \leq \frac{1}{2}\left(A+\sqrt{A^{2}-4 B}\right)=L .
\end{aligned}
$$

Corollary 2.3. Functions $G_{2}, E$ and $F$ satisfy

$$
\begin{aligned}
m_{2} & \leq G_{2}(t, s) \leq M_{2}, \\
E(t, s) & \leq \frac{e^{L}}{e^{l}-1}, \\
F(t, s) & \leq e^{L},
\end{aligned}
$$

where

$$
m_{2}=\frac{T}{(\exp (L)-1)^{2}}, M_{2}=\frac{T \exp \left(\int_{0}^{T} a_{1}(v) d v\right)}{(\exp (l)-1)^{2}} .
$$

Lemma 2.5 ([15]). Suppose the conditions of Lemma 2.2 hold and $h \in P_{T}$. Then the equation

$$
x^{\prime \prime \prime}(t)+p(t) x^{\prime \prime}(t)+q(t) x^{\prime}(t)+r(t) x(t)=h(t),
$$


has a T-periodic solution. Moreover, the periodic solution can be expressed by

$$
x(t)=\int_{t}^{t+T} G(t, s) h(s) d s
$$

where

$$
G(t, s)=\int_{t}^{t+T} G_{2}(t, \sigma) G_{1}(\sigma, s) d \sigma .
$$

Corollary 2.4. Green's function $G$ satisfies the following properties

$$
\begin{aligned}
G(t+T, s+T) & =G(t, s), G(t, t+T)=G(t, t) \exp (\rho T), \\
\frac{\partial}{\partial t} G(t, s) & =(\exp (-\rho T)-1) G_{1}(t, t) G_{2}(t, s) \\
& -b(t) G(t, s)+\int_{t}^{t+T} F(t, \sigma) G_{1}(\sigma, s) d \sigma, \\
\frac{\partial}{\partial s} G(t, s) & =\rho G(t, s),
\end{aligned}
$$

and

$$
m \leq G(t, s) \leq M
$$

where

$$
m=\frac{T^{2}}{(\exp (l)-1)^{2}(\exp (\rho T)-1)}, \quad M=\frac{T^{2} \exp \left(\rho T+\int_{0}^{T} a(v) d v\right)}{(\exp (l)-1)^{2}(\exp (\rho T)-1)} .
$$

\section{Main Results}

In this section we will study the existence of positive periodic solutions of (1).

Lemma 3.1. Suppose $(h 1)-(h 3)$ and (5) hold. The function $x \in P_{T}$ is a solution of (1) if and only if

$$
x(t)=(\exp (\rho T)-1) G(t, t) g(t, x(t-\tau(t)))
$$

$$
+\int_{t}^{t+T} G(t, s)\{f(s, x(s), x(s-\tau(s)))-\rho g(s, x(s-\tau(s)))\} d s .
$$

Proof. Let $x \in P_{T}$ be a solution of (1). From Lemma 2.5, we have

$$
\begin{aligned}
x(t) & =\int_{t}^{t+T} G(t, s)\left[f(s, x(s), x(s-\tau(s)))+\frac{\partial}{\partial s} g(s, x(s-\tau(s)))\right] d s \\
& =\int_{t}^{t+T} G(t, s) f(s, x(s), x(s-\tau(s))) d s \\
(12) & +\int_{t}^{t+T} G(t, s) \frac{\partial}{\partial s} g(s, x(s-\tau(s))) d s .
\end{aligned}
$$


Performing an integration by parts, we get

$$
\begin{aligned}
& \int_{t}^{t+T} G(t, s) \frac{\partial}{\partial s} g(s, x(s-\tau(s))) d s \\
& =\left.G(t, s) g(s, x(s-\tau(s)))\right|_{t} ^{t+T}-\int_{t}^{t+T}\left[\frac{\partial}{\partial s} G(t, s)\right] g(s, x(s-\tau(s))) d s \\
& =(\exp (\rho T)-1) G(t, t) g(t, x(t-\tau(t)))
\end{aligned}
$$

$$
-\rho \int_{t}^{t+T} G(t, s) g(s, x(s-\tau(s))) d s .
$$

We obtain (11) by substituting (13) in (12). Since each step is reversible, the converse follows easily. This completes the proof.

Define the mapping $H: P_{T} \rightarrow P_{T}$ by

$$
\begin{aligned}
& (H \varphi)(t) \\
& =\int_{t}^{t+T} G(t, s)\{f(s, \varphi(s), \varphi(s-\tau(s)))-\rho g(s, \varphi(s-\tau(s)))\} d s \\
& +(\exp (\rho T)-1) G(t, t) g(t, \varphi(t-\tau(t))) .
\end{aligned}
$$

Note that to apply Krasnoselskii's fixed point theorem we need to construct two mappings, one is a contraction and the other is compact. Therefore, we express (14) as

$$
(H \varphi)(t)=\left(H_{1} \varphi\right)(t)+\left(H_{2} \varphi\right)(t) .
$$

where $H_{1}, H_{2}: P_{T} \rightarrow P_{T}$ are given by

$$
\begin{aligned}
& \left(H_{1} \varphi\right)(t) \\
& =\int_{t}^{t+T} G(t, s)\{f(s, \varphi(s), \varphi(s-\tau(s)))-\rho g(s, \varphi(s-\tau(s)))\} d s,
\end{aligned}
$$

and

$$
\left(H_{2} \varphi\right)(t)=(\exp (\rho T)-1) G(t, t) g(t, \varphi(t-\tau(t))) .
$$

To simplify notations, we introduce the constants

$$
\beta=\max _{t \in[0, T]}\{b(t)\}, \delta=\frac{\exp (L)}{\exp (l)-1}, \gamma=\exp (\rho T)-1 .
$$

In this section we obtain the existence of a positive periodic solution of (1) by considering the two cases; $g(t, x) \geq 0$ and $g(t, x) \leq 0$ for all $t \in \mathbb{R}$. For a non-negative constant $\mathbf{K}$ and a positive constant $\mathbf{L}$ we define the set

$$
\mathbb{D}=\left\{\varphi \in P_{T}: \mathbf{K} \leq \varphi \leq \mathbf{L}\right\},
$$

which is a closed convex and bounded subset of the Banach space $P_{T}$. We assume that the function $g(t, x)$ is locally Lipschitz continuous in $x$. That 
is, there exists a positive constant $k$ such that

$$
|g(t, x)-g(t, y)| \leq k\|x-y\|, \text { for all } t \in[0, T], x, y \in \mathbb{D} .
$$

In case $g(t, x) \geq 0$, we assume that there exist a nonnegative constant $k_{1}$ and a positive constant $k_{1}$ such that

$$
\begin{gathered}
k_{1} x \leq g(t, x) \leq k_{2} x, \text { for all } t \in[0, T], x \in \mathbb{D}, \\
\qquad k_{2} M \gamma<1,
\end{gathered}
$$

and for all $t \in[0, T], x, y \in \mathbb{D}$

$$
\frac{\mathbf{K}\left(1-k_{1} m \gamma\right)}{m T} \leq f(t, x, y)-\rho g(t, y) \leq \frac{\mathbf{L}\left(1-k_{2} M \gamma\right)}{M T} .
$$

Lemma 3.2. Suppose $(h 1)-(h 3),(5),(8)$ and (19)-(21) hold. Then $H_{1}$ : $\mathbb{D} \rightarrow P_{T}$ is compact.

Proof. Let $H_{1}$ be defined by (15). Obviously, $H_{1} \varphi$ is continuous and it is easy to show that $\left(H_{1} \varphi\right)(t+T)=\left(H_{1} \varphi\right)(t)$. For $t \in[0, T]$ and for $\varphi \in \mathbb{D}$, we have

$$
\begin{aligned}
& \left|\left(H_{1} \varphi\right)(t)\right| \\
& =\left|\int_{t}^{t+T} G(t, s)\{f(s, \varphi(s), \varphi(s-\tau(s)))-\rho g(s, \varphi(s-\tau(s)))\} d s\right| \\
& \leq M T \frac{\mathbf{L}\left(1-k_{2} M \gamma\right)}{M T}=\mathbf{L}\left(1-k_{2} M \gamma\right) .
\end{aligned}
$$

Thus from the estimation of $\left|\left(H_{1} \varphi\right)(t)\right|$ we have

$$
\left\|H_{1} \varphi\right\| \leq \mathbf{L}\left(1-k_{2} M \gamma\right) \text {. }
$$

This shows that $H_{1}(\mathbb{D})$ is uniformly bounded.

To show that $H_{1}(\mathbb{D})$ is equicontinuous, let $\varphi_{n} \in \mathbb{D}$, where $n$ is a positive integer. Next we calculate $\frac{d}{d t}\left(H_{1} \varphi_{n}\right)(t)$ and show that it is uniformly bounded. By using $(h 1),(h 2)$ and $(h 3)$ we obtain by taking the derivative in (15) that

$$
\begin{aligned}
& \frac{d}{d t}\left(H_{1} \varphi_{n}\right)(t) \\
& =\int_{t}^{t+T}\left[(\exp (-\rho T)-1) G_{1}(t, t) G_{2}(t, s)-b(t) G(t, s)\right. \\
& \left.+\int_{t}^{t+T} F(t, \sigma) G_{1}(\sigma, s) d \sigma\right] \\
& \times\left[f\left(s, \varphi_{n}(s), \varphi_{n}(s-\tau(s))\right)-\rho g\left(s, \varphi_{n}(s-\tau(s))\right)\right] d s .
\end{aligned}
$$

Consequently, by invoking (17) and (21), we obtain

$$
\left|\frac{d}{d t}\left(H_{1} \varphi_{n}\right)(t)\right| \leq
$$




$$
\leq\left[(1-\exp (-\rho T)) M_{1} M_{2}+M \beta+M_{1} \delta T\right] \frac{\mathbf{L}\left(1-k_{2} M \gamma\right)}{M} \leq D,
$$

for some positive constant $D$. Hence the sequence $\left(H_{1} \varphi_{n}\right)$ is equicontinuous. The Ascoli-Arzela theorem implies that a subsequence $\left(H_{1} \varphi_{n_{k}}\right)$ of $\left(H_{1} \varphi_{n}\right)$ converges uniformly to a continuous $T$-periodic function. Thus $H_{1}$ is continuous and $H_{1}(\mathbb{D})$ is contained in a compact subset of $\mathbb{D}$.

Lemma 3.3. Suppose that (18) holds. If $\mathrm{H}_{2}$ is given by (16) with

$$
k \gamma M<1,
$$

then $\mathrm{H}_{2}: \mathbb{D} \rightarrow P_{T}$ is a contraction.

Proof. Let $H_{2}$ be defined by (16). It is easy to show that $\left(H_{2} \varphi\right)(t+T)=$ $\left(H_{2} \varphi\right)(t)$. Let $\varphi, \psi \in \mathbb{D}$, we have

$$
\left\|H_{2} \varphi-H_{2} \psi\right\|=\sup _{t \in[0, T]}\left|\left(H_{2} \varphi\right)(t)-\left(H_{2} \psi\right)(t)\right| \leq k \gamma M\|\varphi-\psi\| .
$$

Hence $H_{2}: \mathbb{D} \rightarrow P_{T}$ is a contraction by $(22)$.

Theorem 3.1. Suppose that conditions $(h 1)-(h 3),(5),(8)$ and (18)-(22) hold. Then equation (1) has a positive $T$-periodic solution $x$ in the subset $\mathbb{D}$.

Proof. By Lemma 3.2, the operator $H_{1}: \mathbb{D} \rightarrow P_{T}$ is compact and continuous. Also, from Lemma 3.3, the operator $H_{2}: \mathbb{D} \rightarrow P_{T}$ is a contraction. Moreover, if $\varphi, \psi \in \mathbb{D}$, we see that

$$
\begin{aligned}
& \left(H_{2} \psi\right)(t)+\left(H_{1} \varphi\right)(t) \\
& =\gamma G(t, t) g(t, \varphi(t-\tau(t))) \\
& +\int_{t}^{t+T} G(t, s)\{f(s, \varphi(s), \varphi(s-\tau(s)))-\rho g(s, \varphi(s-\tau(s)))\} d s \\
& \leq k_{2} M \gamma \mathbf{L}+\mathbf{L}\left(1-k_{2} M \gamma\right)=\mathbf{L} .
\end{aligned}
$$

On the other hand

$$
\begin{aligned}
& \left(H_{2} \psi\right)(t)+\left(H_{1} \varphi\right)(t) \\
& =\gamma G(t, t) g(t, \varphi(t-\tau(t))) \\
& +\int_{t}^{t+T} G(t, s)\{f(s, \varphi(s), \varphi(s-\tau(s)))-\rho g(s, \varphi(s-\tau(s)))\} d s \\
& \geq k_{1} m \gamma \mathbf{K}+\mathbf{K}\left(1-k_{1} m \gamma\right)=\mathbf{K} .
\end{aligned}
$$

This shows that $H_{2} \psi+H_{1} \varphi \in \mathbb{D}$. Clearly, all the Hypotheses of Theorem 1.1 , are satisfied. Thus there exists a fixed point $x \in \mathbb{D}$ such that $x=$ $H_{1} \psi+H_{2} \varphi$. By Lemma 3.1 this fixed point is a solution of (1) and the proof is complete. 
In the case $g(t, x) \leq 0$, we substitute conditions (19)-(21) with the following conditions respectively. We assume that there exist a negative constant $k_{3}$ and a non-positive constant $k_{4}$ such that

$$
\begin{aligned}
k_{3} x \leq g(t, x) \leq & k_{4} x, \text { for all } t \in[0, T], x \in \mathbb{D}, \\
& -k_{3} M \gamma<1,
\end{aligned}
$$

and for all $t \in[0, T], x, y \in \mathbb{D}$

$$
\frac{\mathbf{K}-k_{3} M \gamma \mathbf{L}}{m T} \leq f(t, x, y)-\rho g(t, y) \leq \frac{\mathbf{L}-k_{4} m \gamma \mathbf{K}}{M T} .
$$

Theorem 3.2. Suppose that conditions $(h 1)-(h 3),(5),(8),(18)$ and (22)(25) hold. Then equation (1) has a positive T-periodic solution $x$ in the subset $\mathbb{D}$.

The proof follows along the lines of Theorem 3.1, and hence we omit it.

\section{REFERENCES}

[1] A. Ardjouni, A. Djoudi, Periodic solutions for impulsive neutral dynamic equations with infinite delay on time scales, Kragujevac Journal of Mathematics, 42 (1) (2018), 69-82.

[2] A. Ardjouni, A. Djoudi, Stability for nonlinear neutral integro-differential equations with variable delay, Mathematica Moravica, 19 (2) (2015), 1-18.

[3] A. Ardjouni, A. Djoudi, Stability in nonlinear neutral differential equations with infinite delay, Mathematica Moravica, 18 (2) (2014), 91-103.

[4] A. Ardjouni and A. Djoudi, Existence of periodic solutions for a second-order nonlinear neutral differential equation with variable delay, Palestine Journal of Mathematics, 3 (2) (2014), 191-197.

[5] A. Ardjouni, A. Djoudi, A. Rezaiguia, Existence of positive periodic solutions for two types of third-order nonlinear neutral differential equations with variable delay, Applied Mathematics E-Notes, 14 (2014), 86-96.

[6] A. Ardjouni, A. Djoudi, Periodic solutions for a second order nonlinear neutral functional differential equation with variable delay, Le Matematiche, 69 (2) (2014), 103115 .

[7] A. Ardjouni, A. Djoudi, Existence of positive periodic solutions for a nonlinear neutral differential equations with variable delay, Applied Mathematics E-Notes, 12 (2012), 94-101.

[8] A. Ardjouni, A. Djoudi, Existence of periodic solutions for a second order nonlinear neutral differential equation with functional delay, Electronic Journal of Qualitative Theory of Differential Equations, 2012 (31) (2012), 1-9.

[9] A. Ardjouni, A. Djoudi, Periodic solutions for a second-order nonlinear neutral differential equation with variable delay, Electronic Journal of Differential Equations, 2011 (128) (2011), 1-7. 
[10] A. Ardjouni, A. Djoudi, Periodic solutions in totally nonlinear dynamic equations with functional delay on a time scale, Rendiconti del Seminario Matematico Università e Politecnico di Torino, 68 (4) (2010), 349-359.

[11] T. A. Burton, Liapunov functionals, fixed points and stability by Krasnoselskii's theorem, Nonlinear Studies, 9 (2) (2002), 181-190.

[12] T. A. Burton, Stability by fixed point theory for functional differential equations, Dover Publications, New York, 2006.

[13] F. D. Chen, Positive periodic solutions of neutral Lotka-Volterra system with feedback control, Applied Mathematics and Computation, 162 (3) (2005), 1279-1302.

[14] F. D. Chen, J. L. Shi, Periodicity in a nonlinear predator-prey system with state dependent delays, Acta Mathematicae Applicatae Sinica (English Series), 21 (1) (2005), 49-60.

[15] Z. Cheng, J. Ren, Existence of positive periodic solution for variable-coefficient thirdorder differential equation with singularity, Mathematical Methods in the Applied Sciences, 37 (2014), 2281-2289.

[16] Z. Cheng, Y. Xin, Multiplicity Results for variable-coefficient singular third-order differential equation with a parameter, Abstract and Applied Analysis, (2014), Article ID: 527162, 10 pages.

[17] S. Cheng, G. Zhang, Existence of positive periodic solutions for non-autonomous functional differential equations, Electronic Journal of Differential Equations, (59) (2001), 1-8.

[18] H. Deham, A. Djoudi, Periodic solutions for nonlinear differential equation with functional delay, Georgian Mathematical Journal, 15 (4) (2008), 635-642.

[19] H. Deham, A. Djoudi, Existence of periodic solutions for neutral nonlinear differential equations with variable delay, Electronic Journal of Differential Equations, 2010 (127) (2010), 1-8.

[20] Y. M. Dib, M. R. Maroun, Y. N. Rafoul, Periodicity and stability in neutral nonlinear differential equations with functional delay, Electronic Journal of Differential Equations, 2005 (142) (2005), 1-11.

[21] M. Fan and K. Wang, P. J. Y. Wong, R. P. Agarwal, Periodicity and stability in periodic n-species Lotka-Volterra competition system with feedback controls and deviating arguments, Acta Mathematica Sinica (English Series), 19 (4) (2003), 801-822.

[22] H. I. Freedman, J. Wu, Periodic solutions of single-species models with periodic delay, SIAM Journal on Mathematical Analysis, 23 (1992), 689-701.

[23] H. Gabsi, A. Ardjouni, A. Djoudi, Positive periodic solutions of second-order nonlinear neutral differential equations with variable coefficients, Mathematica Moravica, 22 (2) (2018), 69-82.

[24] M. Gregus, Third order linear differential equations, Reidel, Dordrecht, 1987.

[25] R. Khemis, A. Ardjouni, A. Djoudi, Existence of periodic solutions for a second-order nonlinear neutral differential equation by the Krasnoselskii's fixed point technique, Le Matematiche, 72 (1) (2017), 145-156.

[26] Y. Kuang, Delay differential equations with application in population dynamics, Academic Press, New York, 1993. 
[27] W. G. Li, Z. H. Shen, An constructive proof of the existence Theorem for periodic solutions of Duffng equations, Chinese Science Bulletin, 42 (1997), 1591-1595.

[28] Y. Liu, W. Ge, Positive periodic solutions of nonlinear Duffing equations with delay and variable coefficients, Tamsui Oxford Journal of Information and Mathematical Sciences, 20 (2004), 235-255.

[29] J. Ren, S. Siegmund, Y. Chen, Positive periodic solutions for third-order nonlinear differential equations, Electronic Journal of Differential Equation, 2011 (66) (2011), $1-19$.

[30] D. R. Smart, Fixed points theorems, Cambridge University Press, Cambridge, 1980.

[31] Q. Wang, Positive periodic solutions of neutral delay equations (in Chinese), Acta Mathematica Sinica (N.S.), 6 (1996), 789-795.

[32] Y. Wang, H. Lian, W. Ge, Periodic solutions for a second order nonlinear functional differential equation, Applied Mathematics Letters, 20 (2007), 110-115.

[33] W. Zeng, Almost periodic solutions for nonlinear Duffing equations, Acta Mathematica Sinica (N.S.), 13 (1997), 373-380.

[34] G. Zhang, S. Cheng, Positive periodic solutions of non autonomous functional differential equations depending on a parameter, Abstract and Applied Analysis, 7 (2002), 279-286.

\author{
Abdelouaheb Ardjouni \\ Department of Mathematics and Informatics \\ University of Souk Ahras \\ P.O. Box 1553, Souk Ahras \\ Algeria \\ E-mail address: abd_ardjouni@yahoo.fr
}

\author{
AhCene Djoudi \\ Department of Mathematics \\ University of AnNabA \\ P.O. Box 12, ANNABA \\ Algeria \\ E-mail address: adjoudi@yahoo.com
}

